\title{
Primera aproximación al registro arqueológico del sector oriental del valle de San Antonio de los Cobres, Puna de la provincia de Salta (Argentina)
}

Federico Restifo" y Javier Piraino**

\section{Resumen}

Se presentan los primeros resultados de la investigación arqueológica sistemática llevada a cabo en el sector este del valle de San Antonio de los Cobres. Mediante un relevamiento del registro de superficie a través de transectas, se caracterizó la diversidad del material arqueológico hallado y la cronología relativa. Se recuperó un conjunto lítico y un conjunto cerámico y se identificaron estructuras. La comparación de los materiales hallados con aquellos recuperados en el sector occidental del valle, así como en otras áreas de la Puna de Salta y de la Puna Argentina, sugiere una cronología de ocupación extendida desde el Holoceno temprano. Esto constituye una base de información para continuar las investigaciones.

Preliminary results from the archaeological record of the eastern sector of the San Antonio de los Cobres Valley, Puna of the Salta Province (Argentina)

\begin{abstract}
Here we present the first results of a systematic investigation into the archaeology of the Eastern sector of the San Antonio de los Cobres Valley. We conducted a surface survey using transects, subsequently categorizing the archaeological finds and establishing a relative chronology of the material uncovered. A lithic and a ceramic assemblage were recovered, and structures were identified. The comparison of these materials against those from the western sector of the valley, as well as from other areas of the Puna of Salta and Argentine Puna, suggests an occupational timeline that extends from the early Holocene. This data provides the basis for future research.
\end{abstract}

* Instituto de Investigaciones en Ciencias Sociales y Humanidades (ICSOH), Universidad Nacional de Salta (UNSa) CONICET. Av. Bolivia 5150 (CP 4400) Salta, Argentina. E-mail: federicorestifo@gmail.com

** Facultad de Humanidades, Universidad Nacional de Salta (UNSa). Av. Bolivia 5150 (CP 4400) Salta, Argentina. E-mail: javierpiraino@gmail.com 


\section{Introducción}

El valle de San Antonio de los Cobres (valle de SAC de aquí en adelante), ubicado en la Puna de la provincia de Salta (Figura 1), abarca un área de unos $1.500 \mathrm{~km}^{2}$. Allí se realizan investigaciones arqueológicas sistemáticas al menos desde inicios de la década del '90 del siglo XX (Muscio, 2011). Las mismas se concentraron en el sector occidental y dieron cuenta de una secuencia de ocupación humana que se extiende en el lapso ca. 5300-800 AP, dados los fechados absolutos (Muscio, 2011). Por su parte, el sector oriental sólo fue visitado ocasionalmente, sin desarrollarse trabajos sistemáticos, pero observándose, sin embargo, potencial arqueológico.

En este marco y siguiendo el objetivo general de profundizar la investigación arqueológica en toda la amplitud del valle de SAC, en el mes de marzo de 2016 se inició el trabajo arqueológico sistemático en el sector oriental. La labor realizada privilegió el relevamiento del registro de superficie. En consecuencia, este trabajo se enfoca en presentar los primeros resultados de investigación. Se plantearon dos objetivos particulares: 1) caracterizar la diversidad del registro arqueológico del espacio relevado; y 2) obtener indicadores relativos de la profundidad temporal de la presencia humana en el área a partir de la identificación de artefactos temporalmente diagnósticos.

De acuerdo con estos objetivos se aplicó una metodología centrada en el trazado de transectas en los sectores de fondo de cuenca y quebradas. En este trabajo se presentan los resultados obtenidos a partir del registro arqueológico allí relevado. A su vez, se planteó una discusión de los hallazgos considerando las investigaciones previas en el valle de SAC así como en otras áreas aledañas de la Puna de Salta, como la Cuenca de Pastos Grandes (López, 2009), las que pueden contribuir a estimar la temporalidad de los hallazgos en el área en cuestión.

\section{El sector oriental del valle de SAC en el ámbito general de la Puna Argentina}

La Puna Argentina abarca tierras altas por encima de los $3.000 \mathrm{~m}$ s.n.m. En este ámbito, el valle de SAC se ubica en un sector de transición entre Puna seca y Puna salada (Muscio, 2011). Esta última, ubicada hacia el sur, se caracteriza por ser más fría y árida y con muy bajas precipitaciones que promedian los $100 \mathrm{~mm}$ anuales (Troll, 1958). En términos geológicos, en el valle de SAC se destacan tres complejos principales que estructuran el paisaje: 1) los cordones montanos: comprenden principalmente la Cordillera Oriental (límite oeste del valle) y el conjunto de cerros que forman el límite este del valle de SAC, con cumbres que superan los 5.000 m s.n.m.; 2) las quebradas laterales: bajas y estrechas, recorren transversalmente los cordones montanos presentando cursos de agua permanente; y 3 ) el fondo de cuenca: ubicado en el tramo intermedio del valle, entre las líneas de macizos, se encuentra atravesado en sentido sur-norte por el río San Antonio de los Cobres, lo que constituye también una fuente de agua permanente. En términos arqueológicos, tanto el sector de quebradas laterales como el fondo de cuenca son los más relevantes, ya que constituyen los ambientes de mayor potencial para ocupaciones humanas permanentes (Muscio, 2011).

Particularmente, el sector oriental del valle de SAC (Figura 1) se ubica a unos $15 \mathrm{~km}$ del pueblo de San Antonio de los Cobres. Su extensión aproximada es de $300 \mathrm{~km}^{2}$ y su altura sobre el nivel del mar promedia los $4.000 \mathrm{~m}$. Hacia el Sur está delimitado por la ruta nacional 51, mientras que hacia el Norte por un cordón montañoso que supera los $4.000 \mathrm{~m}$ s.n.m. Hacia el Oeste está delimitado por un conjunto de serranías bajas, las que a su vez definen el límite con el sector occidental del valle. Por su parte, el límite este está dado por un cordón montañoso, también con cumbres por encima 


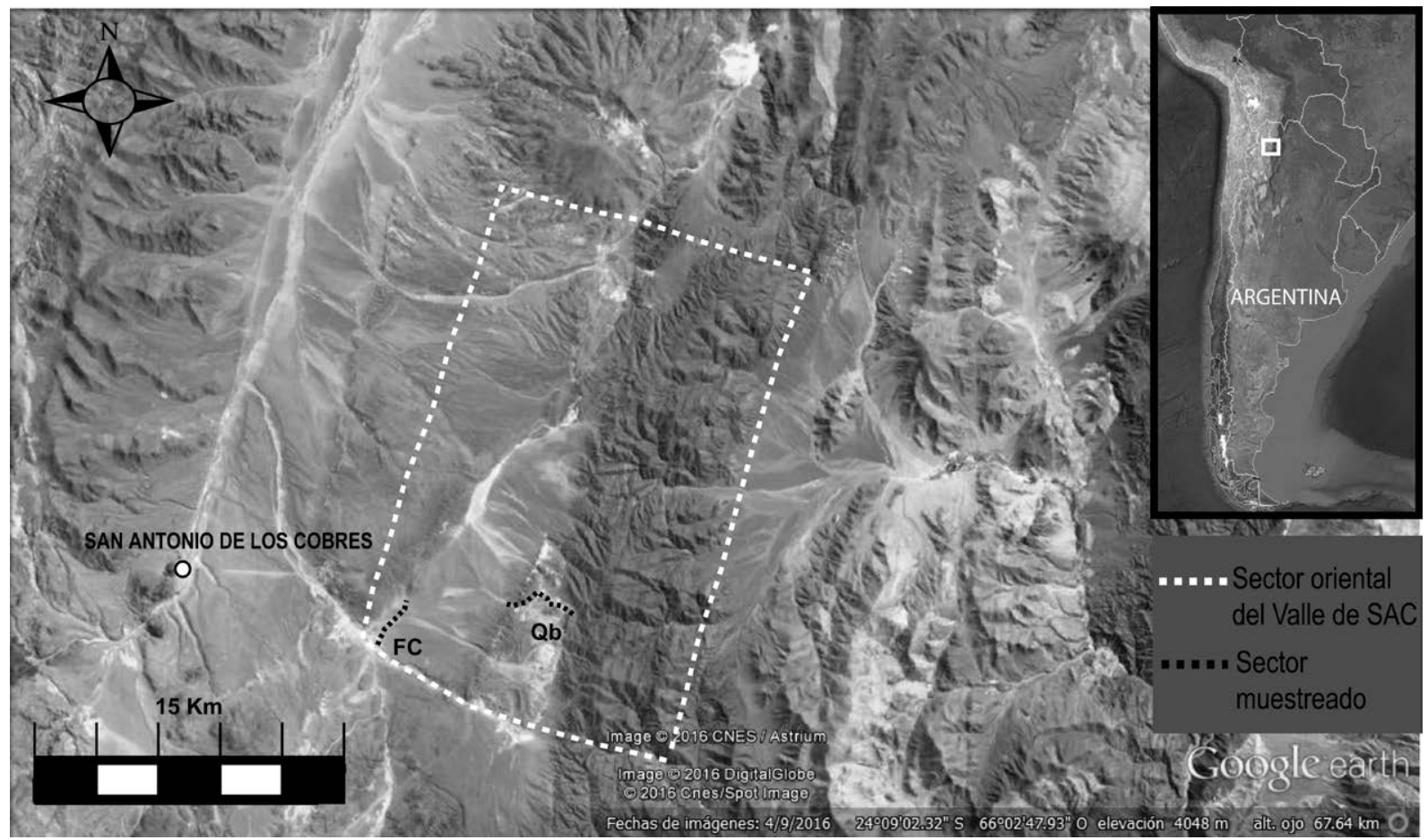

Figura 1: Mapa correspondiente al Valle de San Antonio de los Cobres, con detalle del sector oriental y el espacio muestreado. Referencias: FC: Fondo de cuenca; Qb: Quebrada.

de los $4.000 \mathrm{~m}$ s.n.m., el cual, ya por fuera de los límites del área presentada, incluye al nevado de Acay (5.700 m s.n.m.).

\section{Aspectos metodológicos}

Se realizó una primera prospección mediante el trazado de 18 transectas paralelas, orientadas mediante el uso de GPS y brújula. Se trazaron 12 transectas en el sector de fondo de cuenca, mientras que las seis restantes se plantearon en el sector de quebradas (Figura 1). Las transectas del fondo de cuenca se realizaron de manera lineal, en dirección noroeste, partiendo desde la ruta nacional 51. La extensión de cada transecta fue de $3.000 \mathrm{~m}$ separadas entre sí por 20 metros. A su vez, las mismas fueron divididas en tramos de $100 \mathrm{~m}$, a fin de relevar las frecuencias de material arqueológico en cada una de ellas y poder documentar, con detalle, la variabilidad de la dispersión espacial de este. En cada tramo se relevó el material observable a una distancia de 1,5 m hacia cada uno de los lados.

En relación al sector de quebradas, se realizó el relevamiento en una de ellas (ver Figura 1). En este caso, la extensión de las transectas fue variable. Esto se debió a la estrechez de las quebradas en relación al fondo de cuenca, así como a su curso relativamente sinuoso e irregularidades del relieve, que impidieron la continuidad de las transectas en algunos casos. Por este motivo, también, la separación entre transectas fue menor que en el fondo de cuenca, quedando separadas por $5 \mathrm{~m}$ cada una de ellas. Tres de las transectas pudieron extenderse hasta $2.000 \mathrm{~m}$, mientras que las tres restantes alcanzaron los $3.000 \mathrm{~m}$ proyectados desde el inicio. Se mantuvo la observación de material a 1,5 $\mathrm{m}$ hacia cada lado y la segmentación de las transectas en tramos de $100 \mathrm{~m}$.

Durante el recorrido de las transectas se levantaron los artefactos arqueológicos visibles y en los casos en que se observaron nodos de alta densidad de material, o sitios (Dunnell 
y Dancey, 1983), se procedió a la obtención de sus coordenadas mediante GPS. Los artefactos recolectados se clasificaron de manera general en lítico, cerámica y estructuras arquitectónicas. En relación a la cuantificación, se obtuvieron las frecuencias absolutas y relativas de materiales por cada transecta así como las densidades de materiales en cada una de ellas (Muscio, 2011).

Por otra parte, la cronología se abordó a través de la comparación de los materiales hallados con aquellos artefactos asociados a fechados absolutos en la arqueología de la región y más allá de la misma. En este sentido, la arqueología de la Puna de Salta, así como de otras áreas de la Puna Argentina, ha dado cuenta de contextos arqueológicos estratificados con fechados absolutos que representan diferentes lapsos del Holoceno (López, 2009; Muscio, 2011).

\section{Resultados}

En la tabla 1 se presentan los datos del relevamiento realizado. El sector de quebradas presenta las mayores frecuencias de artefactos, lo que también se refleja en una media de mayor valor $(\overline{\mathrm{X}}=25,33 ; \sigma=50,44)$ respecto de la observada en el fondo de cuenca $(\overline{\mathrm{X}}$ $=6,25 ; \sigma=7,11)$. Por su parte, las frecuencias de artefactos por transecta son variables, lo que da un indicio preliminar de una distribución heterogénea. Asimismo, a nivel general se observaron densidades de artefactos bajas, registrándose un valor máximo de 0,014 artefactos por $\mathrm{m}^{2}$, correspondiente a la transecta 5 del sector de quebradas. Este pico máximo de densidad corresponde a una concentración cuya frecuencia de material supera las 120 piezas, lo que se distingue notablemente del resto de las transectas, tanto en el sector de quebradas como en el fondo de cuenca.

Considerando las clases de artefactos detectadas, se observa que el material lítico es el que predomina de manera notable. Le siguen en orden de frecuencias los tiestos cerámicos y las estructuras. A su vez, en el fondo de cuenca sólo se registró un tiesto cerámico (transecta 1), mientras que en el sector de quebradas se registró mayor frecuencia de tiestos (transecta 5), así como la presencia exclusiva de estructuras (transecta 5).

\section{Artefactos líticos}

En el sector de fondo de cuenca se recuperaron núcleos, desechos de talla, artefactos de filo retocado y un ejemplar de punta de proyectil. Entre los núcleos se registraron ejemplares que evidencian la extracción de lascas. Por su parte, los desechos de talla también corresponden a morfologías de lasca.

En relación a la dimensión temporal adquiere relevancia el registro de un fragmento de punta de proyectil con pedúnculo ancho, bordes curvos y base convexilínea (Figura 2A). El mismo presenta una fracción mínima del limbo, del cual no es posible dar precisiones. En la Puna de Salta no se han recuperado ejemplares con la morfología de pedúnculo mencionada. Sin embargo, considerando el marco macrorregional de investigaciones, la morfología de pedúnculo descripta podría corresponder a puntas de proyectil del Holoceno medio, con una cronología de ca. $6200 \mathrm{AP}$, según los hallazgos del sitio Cueva Salamanca 1, en la Puna de Catamarca (Pintar, 2014, p.60).

Tiene también relevancia cronológica, entre los artefactos de filo retocado, el hallazgo de un ejemplar de artefacto Saladillo (Figura 2B). Dicha clase de artefacto posee regularidades tales como una morfología de contorno general lanceolada, sección plano convexa y uso de hojas como forma base (Fernández, 1983; Restifo, 2015). Ejemplares de esta clase artefactual se hallaron en la estratigrafía de los sitios Ramadas Perfil Norte, ubicado a unos $20 \mathrm{~km}$ del sector oriental del valle de SAC y asociados a 


\begin{tabular}{|c|c|c|c|c|c|c|c|c|c|c|c|}
\hline \multirow{2}{*}{ Sector } & \multirow{2}{*}{ Transecta } & \multirow{2}{*}{$\mathrm{N}^{\circ}$ de tramos* } & \multirow{2}{*}{ Área $\left(\mathrm{m}^{2}\right)^{* *}$} & \multirow{2}{*}{$\begin{array}{c}\text { Frec. de } \\
\text { artefactos }\end{array}$} & \multirow{2}{*}{$\begin{array}{l}\text { Densidad } \\
(\text { artef/m²) }\end{array}$} & \multicolumn{2}{|c|}{ Lítico } & \multicolumn{2}{|c|}{ Cerámica } & \multicolumn{2}{|c|}{ Estructuras } \\
\hline & & & & & & $\mathrm{n}$ & $\%$ & $n$ & $\%$ & $\mathrm{n}$ & $\%$ \\
\hline \multirow{13}{*}{$\begin{array}{l}\text { Fondo de } \\
\text { Cuenca }\end{array}$} & 1 & 30 & 9000 & 13 & 0,0014 & 12 & 92 & 1 & 8 & & \\
\hline & 2 & 30 & 9000 & 22 & 0,0024 & 22 & 100 & & & & \\
\hline & 3 & 30 & 9000 & 13 & 0,0014 & 13 & 100 & & & & \\
\hline & 4 & 30 & 9000 & 3 & 0,0003 & 3 & 100 & & & & \\
\hline & 5 & 30 & 9000 & 3 & 0,0003 & 3 & 100 & & & & \\
\hline & 6 & 30 & 9000 & 13 & 0,0014 & 13 & 100 & & & & \\
\hline & 7 & 30 & 9000 & 3 & 0,0003 & 3 & 100 & & & & \\
\hline & 8 & 30 & 9000 & o & 0 & & & & & & \\
\hline & 9 & 30 & 9000 & 1 & 0,0001 & 1 & 100 & & & & \\
\hline & 10 & 30 & 9000 & 1 & 0,0001 & 1 & 100 & & & & \\
\hline & 11 & 30 & 9000 & 2 & 0,0002 & 2 & 100 & & & & \\
\hline & 12 & 30 & 9000 & 1 & 0,0001 & 1 & 100 & & & & \\
\hline & Total & 360 & 108000 & 75 & 0,008 & 74 & 98 & 1 & 2 & & \\
\hline \multirow{8}{*}{ Quebradas } & 1 & 20 & 6000 & 2 & 0,0003 & 2 & 100 & & & & \\
\hline & 2 & 20 & 6000 & 1 & 0,0001 & 1 & 100 & & & & \\
\hline & 3 & 20 & 6000 & $\mathrm{o}$ & 0 & & & & & & \\
\hline & 4 & 30 & 9000 & 1 & 0,0001 & & & 1 & 100 & & \\
\hline & 5 & 30 & 9000 & 127 & 0,014 & 118 & 93 & 3 & 2 & 6 & 5 \\
\hline & 6 & 30 & 9000 & 21 & 0,002 & 11 & 52 & 9 & 43 & 1 & 5 \\
\hline & Total & 150 & 45000 & 152 & 0,0165 & 132 & 87 & 13 & 9 & 7 & 4 \\
\hline & Suma total & 510 & 153000 & 227 & 0,0965 & 206 & 91 & 14 & 6 & 7 & 3 \\
\hline
\end{tabular}

Tabla 1: Datos de relevamiento correspondientes al recorrido de las transectas en Fondo de cuenca y Quebradas. *Cada tramo tuvo una extensión de $100 \mathrm{~m}$. ${ }^{* * *}$ Valores obtenidos a partir de la multiplicación de la longitud de cada transecta por el ancho de las mismas, correspondiente a $3 \mathrm{~m}$ en todos los casos.

fechados de ca. 5300 años AP, correspondientes a finales del Holoceno medio (Restifo, 2015). También fueron hallados en la capa F2 del sitio Alero Cuevas fechada en ca. 5100-4200 años AP (López y Restifo, 2012). Asimismo, se destaca una fecha de $c a$. 5500 años AP para el sitio Río Grande, en la Puna de Jujuy (Fernández, 1983). Dada esta coherencia de fechados asociados, los artefactos Saladillo pueden considerarse como diagnósticos de tiempo, específicamente del segmento de finales del Holoceno medio (López y Restifo, 2012).

Por su parte, en el sector de quebradas se registraron núcleos, desechos de talla, artefactos de filo retocado, puntas de proyectil y piezas de filo natural con rastros complementarios. Los núcleos hallados evidencian la extracción de lascas, al igual que en el caso del sector de fondo de cuenca. Sin embargo, y a diferencia de este último, en el sector de quebradas se hallaron ejemplares de hojas y de desechos de talla vinculados con la talla laminar (Figura 2C y D). Desde el punto de vista cronológico estos hallazgos adquieren relevancia dado que las hojas se registran en la Puna desde finales del Holoceno temprano, a modo de ejemplares aislados (Hoguin, 2014; Restifo, 2015) y en frecuencias crecientes hacia el momento de finales del Holoceno medio, asociadas a los mencionados artefactos Saladillo (Hoguin, 2014; López y Restifo, 2012; Restifo, 2015). De este modo, la presencia de hojas puede ser un indicador temporal relativo que abarca finales del Holoceno temprano y Holoceno medio.

Entre los hallazgos en el sector de quebradas también se cuentan dos ejemplares de artefactos Saladillo, representativos, tal como se mencionó, de finales del Holoceno 

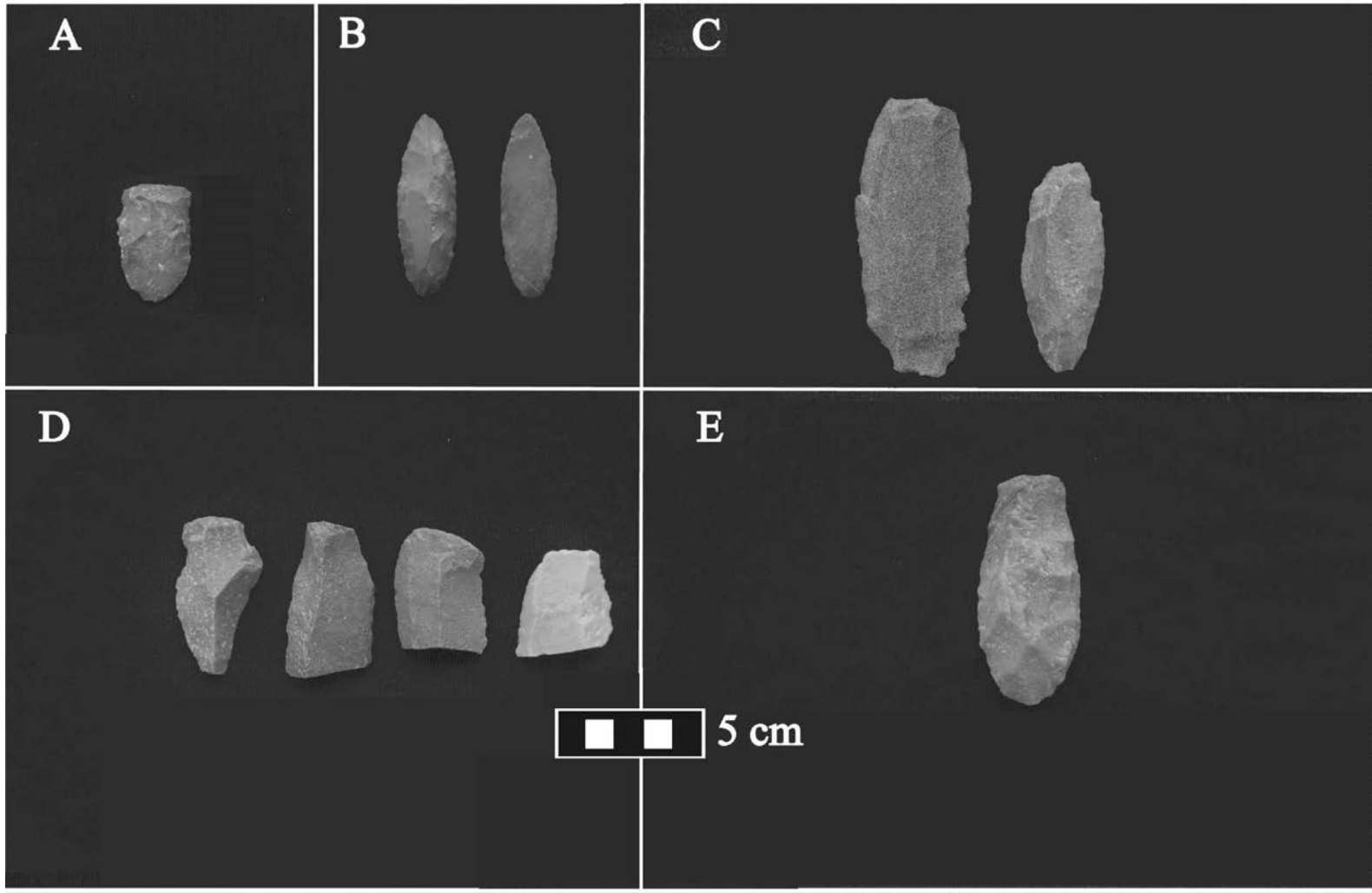

Figura 2: Diversidad de artefactos líticos recuperados a lo largo de las transectas. Ver texto para su descripción.

medio. A su vez, se destaca el hallazgo de un ejemplar de punta de proyectil lanceolada grande (Figura 2E). Esta clase de artefacto es característica de momentos de finales del Holoceno temprano e inicios del Holoceno medio en la Puna Argentina, de acuerdo con lo observado en sitios estratificados de la microrregión de Antofagasta de la Sierra, en la provincia de Catamarca, así como en la Puna de Jujuy (Aschero, 1984; Martínez, 2003).

\section{Cerámica}

Se hallaron 15 fragmentos de cerámica. Sólo uno de ellos fue recuperado en el fondo de cuenca y corresponde a una base plana. El mismo mide $80 \mathrm{~mm}$ de ancho por 60 $\mathrm{mm}$ de largo y presenta un espesor máximo de $12 \mathrm{~mm}$. Se trata de un fragmento sin decoración, en el que se observó una manufactura tosca. Su asignación temporal es dudosa ya que resulta difícil asociarlo a los estilos cerámicos definidos en la región.

Por su parte, se destaca que 12 de los fragmentos restantes fueron recuperados en la transecta 5 del sector de quebradas, en el nodo de alta densidad de material ya mencionado, mientras que los otros dos se recuperaron en el final del último tramo de dicha transecta. En general, se trata de fragmentos pequeños que en promedio miden $20 \mathrm{~mm}$ de ancho por $20 \mathrm{~mm}$ de largo (Castellanos, 2016). Se observó presencia de decoración en cinco casos. Tres de ellos presentan una fina capa de engobe, mientras que en los dos casos restantes se observó pintura sobre la superficie. Estos dos últimos casos resultan interesantes ya que presentan similitudes con el estilo Casabindo, uno de los estilos cerámicos definidos en la región. El mismo corresponde al período Tardío o de Desarrollos Regionales (Albeck y Ruiz, 2003). Se trata de dos fragmentos de cuerpo. Uno de ellos presenta la superficie interna alisada de manera uniforme y la externa con pintura roja y pulido. El espesor máximo es de $6 \mathrm{~mm}$. El segundo fragmento presenta en la superficie externa decoración bicolor consistente en tres líneas paralelas negras sobre un fondo rojo y presencia de 


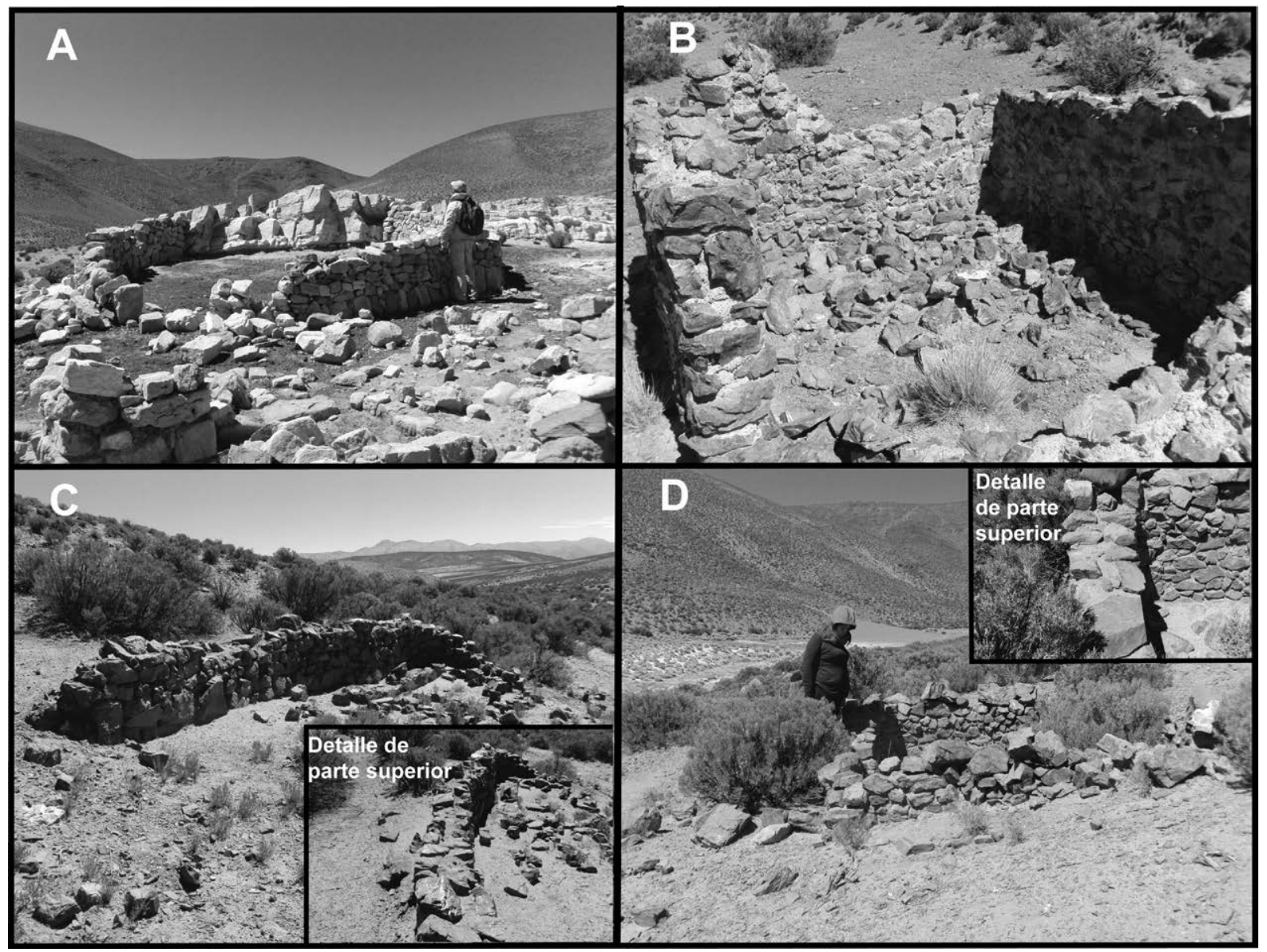

Figura 3: A: Estructura de planta circular, muro de $90 \mathrm{~cm}$ de altura máxima. B: Estructura aislada, muro de 1,20 m de altura máxima. C: Muro, altura máxima de $90 \mathrm{~cm}$. D: Estructura de planta rectangular, muro de $80 \mathrm{~cm}$ de altura máxima.

pulido fino. Por su parte, la superficie interna se encuentra alisada de manera uniforme. El espesor máximo es también de $6 \mathrm{~mm}$.

\section{Estructuras}

Las ocho estructuras detectadas corresponden al sector de quebradas. Las mismas pueden ordenarse en dos subgrupos separados unos $200 \mathrm{~m}$ uno de otro y una estructura aislada separada por una distancia de 1,5 km aproximadamente. El primer grupo está compuesto por cuatro estructuras ubicadas sobre la ladera, las cuales están construidas con rocas de disponibilidad inmediata. Una de las estructuras presenta planta circular (Figura 3A) con un diámetro aproximado de 5 metros. Está construida a partir de una única hilera de rocas, sin argamasa y aprovechando un afloramiento natural como parte de la construcción. A juzgar por la presencia de guano en el interior puede pensarse en su uso como corral. Si bien dicho uso puede considerarse reciente, no se descarta una mayor antigüedad de la estructura. A su vez, a escasos metros de la misma se observaron otras tres estructuras, esta vez de planta cuadrangular y con longitudes de unos 2,5 metros. Las mismas están construidas con la misma roca que la estructura circular, pero a diferencia de esta presentan argamasa, a la vez que se observaron derrumbes. Particularmente, una de ellas presenta dos muros opuestos con terminación triangular, configurando la superficie adecuada para un techo a dos aguas.

El segundo subgrupo está compuesto por dos estructuras, que al igual que el subgrupo anterior se ubican sobre la ladera. Estas fueron construidas con una roca de tonalidad 
gris oscuro, también disponible en las cercanías. Se trata en este caso de un muro (Figura 3C) y una estructura de planta cuadrangular (Figura 3D). El muro posee una longitud máxima de $4 \mathrm{~m}$. Está dispuesto en línea recta y su construcción es en doble hilera con relleno. A unos diez metros del muro descripto se observó la estructura de planta cuadrangular, la cual conserva sus cuatro muros dispuestos en línea recta. Su longitud es de $2 \mathrm{~m}$. Los muros de la estructura en cuestión siguen un patrón de doble hilera.

Por su parte, la estructura aislada (Figura 3B) presenta planta cuadrangular de unos 2 $\mathrm{m}$ de longitud y una construcción en base a muros de doble hilera. También presenta argamasa en las uniones de los bloques de roca. En las cercanías de la misma se hallaron tiestos cerámicos que integran el conjunto descripto en el apartado anterior, entre ellos los fragmentos asignados al estilo Casabindo. Esta estructura es la única en cuyas inmediaciones se hallaron materiales arqueológicos. Para el caso de las restantes, integradas en los dos subgrupos presentados, no se registraron hallazgos en las cercanías. A su vez, considerando todas las estructuras mencionadas, no se registraron hallazgos en su interior.

Desde el punto de vista de la cronología relativa, se considera que por el momento sólo puede hacerse una asignación temporal de alta generalidad. De este modo, las estructuras descriptas reflejarían una temporalidad correspondiente al Holoceno tardío. En este sentido, en el sector occidental del valle de SAC, la fecha más temprana para sitios con presencia de estructuras se remonta a los $c a .2000$ años AP, en la quebrada de Matancillas. Estas se caracterizan por presentar un patrón de planta circular o semicircular (Muscio, 2011). También se registraron hallazgos de conjuntos de estructuras similares en la quebrada de Urcuro y en el sitio Quebrada Alta, este último en la Cuenca de Pastos Grandes, ambos con fechas de ca. 1500 años AP (López, 2009; Muscio, 2011). Las fechas más tardías corresponden al sitio Abra de Minas en la Cuenca del Salar de Ratones, donde se detectó un conjunto de más de 90 estructuras. Las mismas presentan un patrón de planta rectangular y fechados entre $c a$. 680-230 años AP (López y Coloca, 2015), correspondientes al período Tardío-Inca así como a períodos post-hispánicos.

\section{Consideraciones finales}

Si bien la densidad artefactual en relación al espacio relevado es baja, pudieron hallarse materiales arqueológicos diversos. Se destacan tres clases principales: artefactos líticos, cerámica y estructuras. Entre los artefactos líticos las clases con potencial para cronología relativa fueron los desechos de talla laminar, así como las puntas de proyectil y los artefactos Saladillo. Estas clases, en conjunto, representan temporalidades del Holoceno temprano y medio.

Por su parte, el Holoceno tardío está representado por la presencia de cerámica y estructuras. El reconocimiento de dos tiestos cerámicos asignables al estilo Casabindo sugiere una temporalidad correspondiente al período de Desarrollos Regionales o Tardío. En relación a la arquitectura, se plantea su correspondencia con el Holoceno tardío, dados los hallazgos en el valle de SAC, la cuenca de Pastos Grandes y la cuenca del Salar de Ratones, con fechas entre ca. 2000-230 años AP. Si bien en las estructuras relevadas predomina un patrón de planta cuadrangular, el cual es recurrente en el período de Desarrollos Regionales (Albeck y Ruiz, 2003), debe tenerse en cuenta que dicho patrón corresponde a poblados con numerosas estructuras, mientras que en el caso que se presenta en este trabajo se trata apenas de ocho estructuras dispersas a lo largo de unos $3.000 \mathrm{~m}$ de recorrido en transectas. Por este motivo se prefiere plantear una cronología relativa de alta generalidad. Futuras etapas de trabajo como la 
excavación y obtención de fechados absolutos brindarán un panorama más detallado de la cronología.

En resumen, el primer abordaje de la diversidad arqueológica del sector oriental del valle de SAC refleja una cronología relativa de presencia humana de larga data en el área, remontándose al menos hacia finales del Holoceno temprano. Esto sería un hecho factible dadas las características del valle de SAC, con presencia de fuentes de agua permanente y disponibilidad de camélidos, recurso alimenticio fundamental en la Puna (López, 2009; Yacobaccio, 2001). Los resultados presentados, si bien son preliminares, constituyen una base para dar continuidad a los trabajos arqueológicos en el sector oriental del valle de SAC. En este sentido se proyecta la extensión de los muestreos en la dimensión espacial, así como la realización de excavaciones que permitan acceder al conocimiento de la secuencia de ocupación del área y la obtención de fechados absolutos. En última instancia, la ampliación de las investigaciones en el territorio del valle de SAC será relevante para profundizar el conocimiento sobre la historia de ocupación humana de este espacio particular de la Puna Argentina y su evolución a lo largo del tiempo.

\section{Agradecimientos}

Este trabajo contó con el apoyo del proyecto PICT 0320-2013 (Agencia Nacional de Promoción Científica y Tecnológica): "Arqueología de las cuencas de Pocitos y Pastos Grandes, Puna de Salta, Argentina: Procesos de intensificación y domesticación de camélidos, cambio tecnológico y uso del espacio (ca. 10000 años AP - 3500 años AP)", dirigido por el Dr. Gabriel López. Agradecemos al CONICET y a Mirta Santoni, directora del Museo Antropológico de Salta. A Rossana Ledesma, Claudia Subelza, Silvia Soria, Cecilia Castellanos, Jimena Villarroel y Pablo Mignone, colegas de la Universidad Nacional de Salta, por compartir su conocimiento sobre cerámica y arquitectura antigua. A Silvina Seguí, Sonia Araya, Lucía Rucci y Lara Candia Sainz por su valiosa colaboración en el trabajo de campo. Un agradecimiento especial a Celia y Raúl, por permitir las mejores condiciones de hospedaje en San Antonio de los Cobres. 


\section{Q Referencias citadas}

» Albeck, M. E. y Ruiz, M. S. (2003). El Tardío en la Puna de Jujuy: Poblados, etnias y territorios. Cuadernos de la Facultad de Humanidades y Ciencias Sociales - Universidad Nacional de Jujuy, 20, 199-221.

" Aschero, C. (1984). El sitio Inca cueva 4: Un asentamiento precerámico en la Quebrada de Inca Cueva (Jujuy, Argentina). Estudios Atacameños, 7, 62-72.

"Castellanos, C. (2016). Informe de un conjunto cerámico recuperado en el sector oriental del Valle de San Antonio de los Cobres. Manuscrito inédito.

»Dunnell, R. C. y Dancey, W. S. (1983). The siteless survey: a regional scale data collection strategy. En M. B. Schiffer (Ed.), Advances in Archaeological Method and Theory (Volume 6) (pp. 267-287). Orlando: Academic Press.

» Fernández, J. (1983). Río Grande. Exploración de un centro precerámico en las altas montañas de Jujuy, Argentina. Ampurias, 45-46, 54-83.

» Hoguin, R. (2014). Secuencia cronológica y tecnología lítica en la Puna seca y salada de los Andes Centro-Sur para el Holoceno temprano y medio a través del ejemplo de Susques. Relaciones de la Sociedad Argentina de Antropología, XXXIX(2), 333-364.

" López, G. (2009). Diversidad arqueológica y cambio cultural en Pastos Grandes, Puna de Salta, a lo largo de Holoceno. Relaciones de la Sociedad Argentina de Antropología, XXXIV, 149-175.

"López, G. y Restifo, F. (2012). The Middle Holocene domestication and intensification of camelids in north Argentina, tracked by zooarchaeology and lithics. Antiquity, 86, 10411054.

» López, G.y Coloca, F. (2015). El sitio Abra de Minas: nuevos aportes para la caracterización de las ocupaciones Tardío/Incas en las tierras altas del noroeste argentino. Bulletin de I'Institut Français d'Etudes Andines, 44(1), 141-149.

"Martínez, J. (2003). Ocupaciones humanas tempranas y tecnología de caza en la microrregión de Antofagasta de la Sierra (10000-700o AP). (Tesis Doctoral inédita), Universidad Nacional de Tucumán, Argentina.

» Muscio, H. (2011). Ocupaciones humanas a cielo abierto de finales del Holoceno medio y comienzos del Holoceno tardío en el Valle de San Antonio de los Cobres, Puna de Salta. Comechingonia. Revista de Arqueología, 15, 71-90.

»Pintar, E. (2014). Continuidades e hiatos ocupacionales durante el Holoceno medio en el borde oriental de la Puna salada, Antofagasta de la Sierra, Argentina. Chungara. Revista de Antropología Chilena, 46, 51-71.

»Restifo, F. (2015). Tecnología de hojas líticas en tierras altas andinas: Perspectivas desde la Puna de la Provincia de Salta (Argentina). Estudios Atacameños, 51, $33-51$.

»Troll, C. (1958). Las culturas superiores Andinas y el medio geográfico. Revista del Instituto de Geografía, 5, 3-55.

" Yacobaccio, H. (2001). La domesticación de camélidos en el Noroeste Argentino. En E. E. Berberián y A. E. Nielsen (Eds.), Historia Argentina Prehispánica (Tomo 1) (pp. 7-40). Córdoba: Editorial Brujas. 